\title{
A framework to manage reluctance to bad news reporting on software projects in state universities in Zimbabwe
}

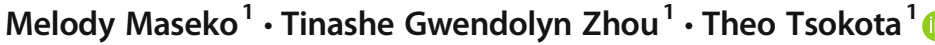

Received: 16 May 2019 / Accepted: 21 May 2020 / Published online: 31 May 2020

(C) Springer Science+Business Media, LLC, part of Springer Nature 2020

\begin{abstract}
Failure of IT projects has risen to become an eyesore for most organisations. This is often attributed to the 'mum effect'- an individual's reluctance to report the exact position of troubled software projects. With the increasing digitalization of operations by most State Universities in Zimbabwe, the mum effect has the potential to rise to exponential proportions. Guided by the Design Science approach data was gathered from an intensity sample of 15 participants comprising 7 software project team members, 3 ICT project managers, and 5 users of the system drawn from three Zimbabwean state universities. The data collection was done using key informant interviews and focus group discussions. Data were analysed using thematic analysis and NVivo (version 11) software was used to store, organise, and code data transcripts. Factors suppressing Bad News Reporting were grouped into three broad categories which included organisational factors, personal factors, and situational factors. The findings suggest the need to incorporate these multi-level factors in the design and implementation of software projects if they are to be efficacious. This study recommends the establishment of clear channels of communication to manage bad news reporting and creating formal structures that function outside the traditional organisational hierarchy to convey information regarding anomalies. This study contributes to practice by providing appropriate interventions based on empirical evidence based on the centrality of software project team members' insights, experiences as well as practices.
\end{abstract}

Keywords Bad news reporting $\cdot$ Software projects $\cdot$ State universities $\cdot$ Zimbabwe . Framework

Theo Tsokota

tsokotat@staff.msu.ac.zw

1 Department of Information System, Midlands State University, P. Bag, 9055 Gweru, Zimbabwe 


\section{Introduction}

The importance of Information and Communication Technology (ICT) in developing both society and individuals cannot be overemphasised (Avgerou \& Walsham 2017). When used appropriately, different ICT help to extend the access of education, strengthen the relevance of education to the increasingly digital workspace, and raise educational quality thus help make teaching and learning an engaging, active process connected to real life (Veletsianos \& Kimmons 2016) Consequently, universities that leverage on ICT have profound concerns about the general success of software projects since they make huge investments in information systems. The failure of software projects continues to be one of the notable challenges despite the visible advances in ICT. This is partly caused by the failure of projects to keep up with a schedule, resulting in some projects going significantly over budget (Keil \& Park 2010; Kliem \& Ludin 2019). This can be attributed to the fact that the process of software is development largely depend on human agency and is thus susceptible to varying risks as well as challenges, especially those associated with human behaviour and mental processes (Teklemariam \& Mnkandla 2017; Ramingwong \& Ramingwong 2013).

The mum effect (an individual's reluctance to report the exact position of failing software projects) is a major reason for the failure of IT projects (Kliem \& Ludin 2019; Keil \& Park 2010; Ramingwong \& Ramingwong 2013). According to Keil \& Park (2010), the reluctance to escalate information on the exact position of troubled software projects to fellow members of the project team has recently received renewed attention as a significant factor contributing to the failure of projects.

Risks such as the mum effect are a result of deliberate decisions by at least one stakeholder to withhold important information as a way of avoiding unpleasant consequences (Ramingwong \& Ramingwong 2013; Zanin et al. 2016). According to Ramingwong \& Ramingwong (2013), people are generally inclined to withhold information about failing projects to responsible authorities. Thus, those in authority often fail to institute corrective measures in time to arrest the situation. Chatterjee \& Maji (2018) caution that it is important for project team members to report the status of troubled projects so that the necessary remedial actions to revive the projects can be taken. Where the project is beyond redemption, the timeous reporting of bad news would allow authorities to even abandon the project. In the same vein, Natovich et al. (2013) remark that if the actual status of a software project is not known, it becomes difficult for project leaders to make critical decisions on whether or not to abandon a project.

The mum effect also affects ICT-intensive institutions like universities where ICT systems are the backbone of their information, data management, and learning infrastructure (von Solms 2006). These establishments have some product ventures in ICT that are experiencing improvements such as e-learning and online learning which are of high importance. These ICT developments are likely to continue particularly in an environment characterised by disruptive global phenomena such as the Covid-19 pandemic. If appropriate status reporting systems are not followed during system development and improvement, universities face the colossal danger of failing to safely coordinate data and ICT as a conveyance medium in teaching, monitoring and evaluation programs (von Solms 2006; Eldow et al. 2017). Like any other department in any institution, the ICT department is a cost center. This makes it imperative that any incidence of bad news in programming activities should be reported on time to minimise costs resulting from project failures. Hesitation to do 
so endangers the development of ICT projects because the cost of undertaking them will shoot over the roof. Consequently, educators and administrators cannot afford to ignore the mum effect. It is this observation that has spurred this drive to come up with a systematic framework to understand the mum effect.

This study set out to develop a framework that spells out a systematic approach to reporting of bad news on failing software projects at Zimbabwean State Universities. The study specifically sought to establish the causes of individuals' inclination to not reporting Bad News on troubled software projects, identify the major drivers for BNR on such projects, spell out the effects of not reporting unpleasant news on troubled software projects and formulate strategies for systematically encouraging BNR on troubled software projects.

This paper is organised into five sections. It will first discuss the theoretical foundations of BNR including the model which informed the research. The research methodology will be discussed thereafter. Then the results will be presented as well as the proposed framework. Lastly, the conclusion of the study will be given.

\section{Literature review}

This section reviews the work that was done by others and highlights the theoretical lens that informed the study.

\subsection{The mum effect}

According to Dibble \& Levine (2010), the mum effect refers to the robust research finding that people are more hesitant to share bad news relative to the good news. These findings support the research by McBride (2016) who concluded that communicators are more biased towards encoding or transmitting to their audience pleasant news and avoid conveying unpleasant news.

There are a number of factors which lead to the mum effect including the fear that merely conveying negative information will enlist negative consequences and information asymmetry (Padhi \& Sumita 2017); culture (Ramingwong \& Ramingwong 2013; Jung-Chieh et al. 2016); real or perceived workplace bullying exhibited by a supervisor against a subordinate (Beakley 2016); the individual may not be completely aware of the degree of the inconvenience notwithstanding noticing that the project is behind calendar (Aronson 2016); risk perception (Kliem \& Ludin 2019); team solidarity (Padhi \& Sumita 2017); fault responsibility and time urgency (Dibble \& Levine 2010); moral intensity (Padhi \& Sumita 2017); IT failure impact and personal morality (Teklemariam \& Mnkandla 2017) or the people may withhold the bad news out of selfconcern, out of concern for the recipient or simply to comply with norms (Tesser \& Rosen 1975) among other factors. All these factors are put into three broad categories namely: personal factors, situational factors and organizational factors.

\subsection{Theoretical model underpinning the study}

This study was grounded on the mum effect model (Smith \& Keil 2003) shown in Fig. 1, which asserts that individuals involved in project development constantly 


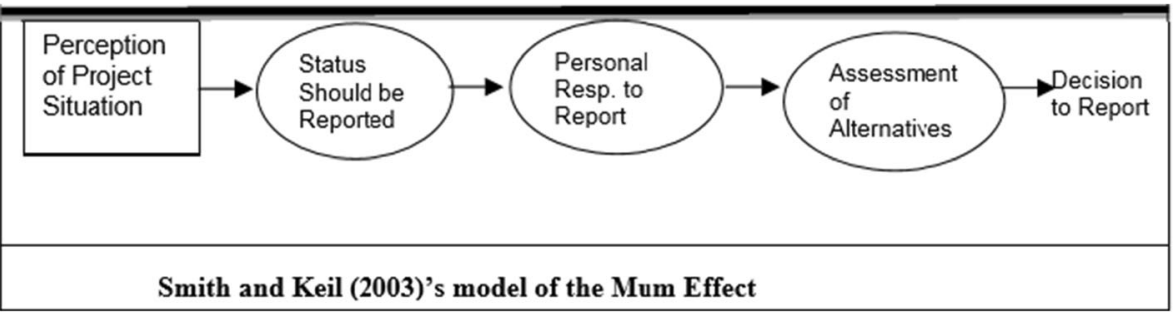

Fig. 1 Mum effect model: (Smith \& Keil 2003)

monitor and evaluate the status of the project. The obligation to dispassionately evaluate the project and therefore report sections and components of the project that may not be performing to expectation is guided by individual moral norms. In any case, the responsibility or commitment to make a report has its challenges. The one who has to make the report has to assess the potential consequences of making or not making that report, especially where it is going to be a negative report. One may consider a possibility of retaliation, being labelled a back-stabber by team members who may be found at fault, and the probable consequences of remaining a group member versus being an outcast. In this way, reporting or not reporting hinges on the individual's judgment regarding the consequences of either action.

This model is one of the fundamental models for BNR. It was chosen for the study because it was developed specifically to explain the reasons why people are reluctant to transmit negative information, particularly within a software project context.

\section{Methodology}

The study utilised the Design Science (DS) research approach as a methodology. This included conducting focus group discussions and key informant interviews. The interpretive paradigm informed the research and therefore qualitative approach was adopted. To unpack the multiple realities attendant to BNR prevalent in endangered software projects, the researchers adopted a subjectivist-transactional epistemological position.

\subsection{Why design science research?}

According to Peffers et al. (2007) design science concerns itself with creating an artefact as a solution to such a problem. Thorough procedures to design and create artefacts to tackle these challenges are the main feature of DS. This involves making research input, evaluating the designs, and communicating the conclusions to exact addressees. These artefacts may include models, instantiations, concepts, and approaches. In this research, software projects were the artefacts meant to solve problems in ICT.

According to Hevner et al. 2004), the artefact must be state-of-the-art, capable of solving a problem or unraveling an identified problem in an effective and resourceful way. In line with this statement, this study did not just set out to deal with troubled software projects in general. It also sought to formulate an innovative and systematic 
framework for BNR, which in its own right would be an artefact that is a valuable addition to software project solutions.

\subsection{Sampling}

The sample for this study was made up of 15 participants comprising 7 software project team members, 3 ICT project managers, and 5 system users drawn from three Zimbabwean state universities. The sample was selected using intensity sampling which involves the selection of information-rich cases that manifest the phenomenon of interest intensely. As such the participants were selected based on them being members of the software project team and user of the system under development. The sample was structured as shown in Table 1.

\subsection{Data collection}

Ten key informant interviews with project team members were conducted for this study. Interviews are a particularly useful instrument in getting the story behind an interviewee's experiences. Through interviews, rich information about individuals' aversion to BNR could be extracted from participants. A focus group discussion was conducted to gather data from five system users. The researchers chose to use focus groups on system users because they were the most appropriate considering the resources available. Hevner \& Chatterjee (2010) state that through focus groups a researcher can get insights into people's thoughts. They also help the researcher get an in-depth understanding of the phenomena under study.

\subsection{Data analysis}

The Braun \& Clarke (2006) method of analysing data was followed. Data was analysed in two phases, that is, structural coding followed by thematic analysis. The themes were developed and identified in the field during the data gathering process and not before the researchers went into the field. As a result, the concepts originated from the interviewee(s) during data collection, only to be developed and further conceptualised by the researchers.

Table 1 Sample distribution table

\begin{tabular}{lll}
\hline Sample & Size & Institution \\
\hline Project managers & 1 & Institution A \\
Project managers & 1 & Institution B \\
Project managers & 1 & Institution C \\
Project team members & 2 & Institution A \\
Project team members & 2 & Institution B \\
Project team members & 3 & Institution C \\
System users & 5 & Institution A \\
\hline
\end{tabular}


In order to allow users to freely divulge as much information as possible about their views on BNR, the interviewer did not ask leading questions. To extract the experiences that were important to the participant(s) the researchers devised and assigned theoretical labels, which are codes. Members of the research team analysed the data independently and met to iron out coding discrepancies. Codes were identified for similarity and were grouped into emergent thematic categories. These were then debated, refined, revised and merged by the research team. A number of these codes were put into abstract groups or categories which eventually formed the basis for theory building. It should be noted that the aim was never to combine information across respondents, but just to uncover similarities and variances across the entire spectrum of voices. To store, organise, and code the transcripts, the researchers used NVivo (version 11) software.

\section{Results and discussion}

This section presents the results and discussion under following subheading organisational, personal and situational factors. It will begin by showing thematic codes that emerged from the data.

The codes in Table 2 were marked to determine the frequency. Once the data was captured, distinct themes were identified, extracted and coded. Multiple iterations ensured that the identified themes were accurate and complete. In situations where different responses pointed to the same aspect, a broad key phrase was identified to provide one common description for the unstructured data. The frequency of each code was tallied and converted into a percentage for each of the categories. From the data collected the marked codes totalled 37 and all the codes had a recurrence that amounted to 321 .

\subsection{Factors inhibiting bad news reporting}

The study sought to establish the factors behind individuals' tendency to hesitate to report bad news. In total, 13 factors that trigger hesitance to report bad news were identified. These were categorised into three broad groups as follows: (a) organisational factors (i.e. policies, organisational structure, culture and frameworks for project status reporting); (b) situational factors (i.e. fault responsibility, time urgency, perceived risk, fear, and uncertainty) and; (c) personal factors (i.e. risk propensity, locus of control, professional expertise, cognitive biases, and past experience).

\subsubsection{Organisational factors}

Under organisational factors, policies appeared to be a very important factor in triggering reluctance to BNR. It had the highest number of tags compared to organisational structure and organisational culture. This study disproves Shaya (2013) findings to the effect that culture is the most important factor inducing the mum effect. This study also reveals that the failure to formulate and implement effective ICT policies has a greater influence on the mum effect. This inconsistency with prior research over factors influencing the mum effect could be a result of the 
Table 2 Thematic codes from the data

\begin{tabular}{|c|c|c|c|}
\hline Ranking & Code label & Number of tags & Cumulative tags \\
\hline 1 & Policies & 14 & 14 \\
\hline 2 & Structure/hierarchy & 3 & 17 \\
\hline 3 & Culture & 2 & 19 \\
\hline 4 & Project status reporting frameworks & 8 & 27 \\
\hline 5 & Risk propensity & 10 & 37 \\
\hline 6 & Locus of control & 5 & 42 \\
\hline 7 & Professional expertise & 12 & 54 \\
\hline 8 & Cognitive biases & 3 & 57 \\
\hline 9 & Fault responsibility & 8 & 65 \\
\hline 10 & Time urgency & 9 & 74 \\
\hline 11 & Understanding team dynamics & 4 & 78 \\
\hline 12 & Information asymmetry & 7 & 85 \\
\hline 13 & Fear and uncertainty & 13 & 98 \\
\hline 14 & Past experiences & 10 & 108 \\
\hline 15 & Rewards & 5 & 113 \\
\hline 16 & Skills deficits & 7 & 120 \\
\hline 17 & Negative motivation & 5 & 125 \\
\hline 18 & Equitable distribution of work & 8 & 133 \\
\hline 19 & Effective group communication & 10 & 143 \\
\hline 20 & Monitoring and evaluation & 5 & 148 \\
\hline 21 & Malfunction in the system & 13 & 161 \\
\hline 22 & Project failure & 15 & 176 \\
\hline 23 & Organisational culture change & 9 & 185 \\
\hline 24 & Feedbacks & 12 & 197 \\
\hline 25 & Delays in project completion & 13 & 210 \\
\hline 26 & Project escalation & 13 & 223 \\
\hline 27 & Punishments & 7 & 230 \\
\hline 28 & The variance between the delivery project and user requirement & 8 & 238 \\
\hline 29 & Lack of trust and user resistance & 9 & 247 \\
\hline 30 & Engagement through meetings & 12 & 259 \\
\hline 31 & Awareness programmes & 8 & 267 \\
\hline 32 & Project management training & 10 & 277 \\
\hline 33 & Achievement motivation & 12 & 289 \\
\hline 34 & Project status reporting policies & 14 & 303 \\
\hline 35 & Ripple effects on business processes & 6 & 309 \\
\hline 36 & Work plan & 5 & 314 \\
\hline 37 & Open door policy & 7 & 321 \\
\hline
\end{tabular}

studies having been carried out in dissimilar industries or environments. Shaya (2013) studied consultancies that were made up of individuals originating from different countries, hence culture played a prominent role. 
According to Natovich et al. (2013), a great deal of BNR research has dwelt on general aspects of the phenomenon such as risk perception (Kliem \& Ludin 2019), time management (Teklemariam \& Mnkandla 2017), organisational culture and information blind-spots (Kliem \& Ludin 2019), the perceived effect of not reporting bad news and the challenges connected to the ICT project. It is in this vein that this research concluded that culture does indeed play a significant role in BNR. Several participants in this study indicated that in Zimbabwean state universities, the prevailing culture does not promote BNR. This is because for an individual to report bad news regarding a troubled software project, they risk being perceived as lacking in skills or ability to rectify the problem themselves. These causes would-be reporters to go mum. In this instance, reporting bad news on projects can lead to the misconception by management threatening individual's jobs. This, therefore, triggers hesitance to give aversive reports on project status.

Thus, in meetings to evaluate processes, project managers tend to cover up their backs than expose themselves and appear as if they are incompetent before their peers and superiors. One participant showed more confidence in reporting bad news because in their institution there was an open door policy and work plans were properly structured and formalised. Ramingwonga \& Snansieng (2013) studied how organisational culture contributes to the mum effect. This study revealed that project team members do not always get feedback after conveying bad news on projects leading to reluctance to tale any future negative news on a project.

\subsubsection{Situational factors}

These are contextual factors that are connected with a particular software project. Research findings have highlighted that factors such as responsibility for fault, time urgency, fear are largely responsible for individuals' reluctance to report bad news about any software project. Jung-Chieh et al. (2016) agree that fault responsibility employs both direct and indirect influence on readiness to report bad news, whereas time urgency was found only to put forth an indirect influence on preparedness to report bad news.

According to Smith \& Keil (2003), four critical situational factors have the potential to affect the perceptions of an ICT project situation. These are risk, time pressure, level of behavioural immorality, and information asymmetry. This study's findings conform to Smith \& Keil (2003) revelations on time pressure as it were. The issue of deadlines regarding software projects has in both studies featured prominently as one of the major factors triggering reluctance to report bad news. As the deadlines approach while a member of the project team is facing challenges to stay within the project time frame, silence becomes the best way to handle the attendant pressure and anxiety.

One critical force that can impact negative conduct in BNR is simply choosing to give a deaf ear to whatever issues are raised regarding the project status. This is known as the 'deaf effect' and participants in this study did allude to it in some way as a key factor. This was apparent when they mentioned a lack of feedback from their superiors. Beakley (2016) points out that, project team members do encounter challenges when their superiors do not respond to project status reports they would have submitted fully expecting guidance, feedback, or some form of response. In this study 12 participants indirectly alluded to the deaf effect. 


\subsubsection{Personal factors}

The literature on BNR posits that affective issues and individual personalities play a critical role in individuals' BNR decision making (Smith \& Keil 2003). In apparent confirmation of this position, Aronson (2016) highlights that personality and communication are innately interrelated. This study also shows a larger number of tags on personal factors Vis-à-vis the other two, that is, organisational and situational factors. This confirms the prominence of its impact on BNR. In this study risk propensity, locus of control, professional expertise, cognitive biases, and past experiences also emerged as some of the major factors in BNR. Professional expertise and risk propensity ranked way above the other three factors. In their study, Padhi \& Sumita (2017) noted that moods do affect individuals' opinions, judgments, and choices. This study posits that a person's mood affects their willingness to account for the errors they would have committed. In this demise, individuals in an optimistic frame of mind will be more prepared to report a self-committed error relative to people in a negative mood.

Teklemariam \& Mnkandla (2017) see risk as an event that happens suddenly without prior warning. It may be a state or condition that arises suddenly at any stage of the project and whose occurrence instigates negative impacts. In this research, risk emerged as a serious issue instigating unwillingness to BNR. Aronson (2016) similarly found some experimental evidence that personal factors like risk propensity do have an effect on the judgement and decision making pertaining to reporting a project's status. This research highlighted that individuals do not have uniform risk behaviours as some are risk-takers, meaning they are not afraid of the outcomes of bad news, others who are risk-averse (those who fear the outcomes of reporting negative news), and yet others who are risk-neutral. The number of personal factors was greater in terms of influencing BNR compared to the other two broad factors. The study also sheds light on the interrelation of these factors, meaning that a certain organisational factor can trigger one or more personal factors that suppress bad news reporting.

\subsection{Major drivers of BNR on troubled software projects}

Eight drivers of BNR include; team dynamics, reward systems, skills deficits, punishment and equitable distribution of work, achievement motivation, effective group communication, and feedback came up from this research. Feedback had more tags than all the other factors, highlighting its dominance in triggering people to report bad news. Equitable distribution was the second-highest in this respect. In an organisational context, feedback pertains to the information sent to individuals or a group regarding their prior behaviour and such information seeks to compel, instruct or persuade the individuals or group to alter their present and future behaviour to get the preferred organisational outcomes. This research does richly contribute to the Information Systems field through the introduction of the mum effect construct, hitherto an uncharted territory, particularly in Zimbabwe.

McBride (2016) emphasises the significance of sustained communication in driving projects to successful completion. In addition, Wixom \& Watson (2001) maintain that the participation of users coupled with the team's skills set are vital implementation factors that have a decisive bearing on the success or failure of a project. Thus, Wixom and Watson (2001) regard these two factors essentially as communication skills. This 
study concurs with the findings of the above-cited authorities regarding the importance of active and effective group communication, the team's skills set, and last but not least feedback, which is also regarded as a component of user involvement. User involvement has a strong bearing on the success story of a project.

Participants emphasised the significance of the equal distribution of workload. The participants pointed out that after one has reported bad news, certain tasks will be added to their workload. Therefore, to avoid that extra load, the project members would rather go mum on any negatives relating to the software project. In support of this study's findings, Milliken et al. (2003) did discover that a common tactic to avert job overload is to deal with the idea that one would be negatively tagged if they dared speak up. According to this study, members of a project often hesitate to report negative news in fear of being labelled incompetent. If disclosure of bad news attracted positive labels like "courageous" rather than contemptuous labels like "troublemaker," the propensity to report would be greater than that of maintaining silence. Using the organisation's code of conduct to classify such negative labels as workplace harassment would certainly help motivate people to report anything of importance on a software project. In light of this, it is important for project managers to understand team dynamics and the meaning of balancing workload so as to come up with more engaged team members.

Some factors specifically pertain to the population under this study and because of this, not much literature on the drivers of BNR could be found. The section on strategy can be regarded as an important addition to the BNR knowledge body. The majority of authors focused on the factors leading to the mum effect without examining the drivers, a gap which this study addressed.

\subsection{Strategies to encourage BNR on troubled software projects}

Strategies that participants raised as recommendations for managing BNR in Zimbabwean state universities include training of project team members, conducting awareness programmes, monitoring, and evaluation, changing the culture of the organisational, as well as ensuring job security and reporting instruments. Interestingly reporting instruments had the highest frequency in this study which means that there is a greater need to formalise project status reporting policies and procedures. A total of 14 participants were of the view that their institutions needed policies that relate to software projects status reporting. Also high on the participants' responses was a change in organisational culture and the need for awareness programmes with 8 tags each.

In a significant contribution to the issue of managing BNR, Milliken et al. (2003) suggested that managers who are serious about designing learning organizations should realise that employees tend to regard being vocal about their concerns as an unworthy risk which may end up backfiring on them. This leads them to keep to themselves any information regarding potential problems or issues. Where management seriously expects project team members to cooperate and speak up about problems they are facing, they need to find ways of convincing organizational members that they not only want to know the problems or issues the employees are facing but also assure them that no one will be victimised thereafter. It is imperative for organisational leaders to create workplace conditions that make workers feel safe to speak their minds (Chatterjee \& 
Maji 2018). This study, however, did not capture this important contribution by Milliken et al. (2003) and Chatterjee \& Maji (2018).

It is Korzaan \& Brooks' (2014) argument that because senior management wields control over the work climate, it is their responsibility to implement policies that promote a culture of open communication, especially pertaining to project status. In this study, 14 of the participants were of the view that the implementation of status reporting policies was an important solution in managing the mum effect amongst project team members.

As suggested by Zanin et al. (2016), executives should guard against the chances of a backlash visiting project team members for reporting negative information. There should be an openness to foster and encourage open communication. This issue was not clearly alluded to in this study as the participants only mentioned the need for favourable policies as a way of managing the mum effect. In this vein, it should be highlighted that State universities by nature have a hierarchical structure that researchers have identified as an impediment to BNR.

The need for senior managers to pay close attention to the make-up of steering committees of projects is another important aspect that Zanin et al. (2016) also highlighted. In this study, this point came up under the feedback tag. Participants constituting key users of the system pointed out that the composition of project steering committees in Zimbabwe's state universities was not balanced because the key users were not represented at that level. This naturally creates negative effects such as user resistance as well lack of trust on the part of the system users.

Kliem \& Ludin (2019) state that team composition is an important factor in instances where cultural differences have to be accommodated particularly in multi-cultural or even multi-racial institutions. There the steering committee needs to be balanced and this helps manage BNR.

\section{Proposed framework to manage bad news reporting in state universities}

From the analysis of gathered data and expert reviews, a model to manage reluctance to BNR in Zimbabwean State Universities was developed and was reviewed by four experts. The next subsection will describe the Framework shown in Fig. 2.

\subsection{Description of the framework}

The key factors behind individuals' tendency to hesitate to report bad news were group under the following headings organisational, situational, and personal factors. In addition, major drivers and strategies to encourage BNR of software projects were identified. These were synthesised to produce a framework that incorporated Smith and Keil's (2003) Mum Effect Model. In the proposed framework factors suppressing and encouraging BNR were added. Thus, the decision to report is a product of the interplay between factors inhibiting and factors encouraging BNR. The framework will now be explained in detail.

According to the proposed framework individuals involved in project development constantly monitor and evaluate the status of the project. The obligation to 


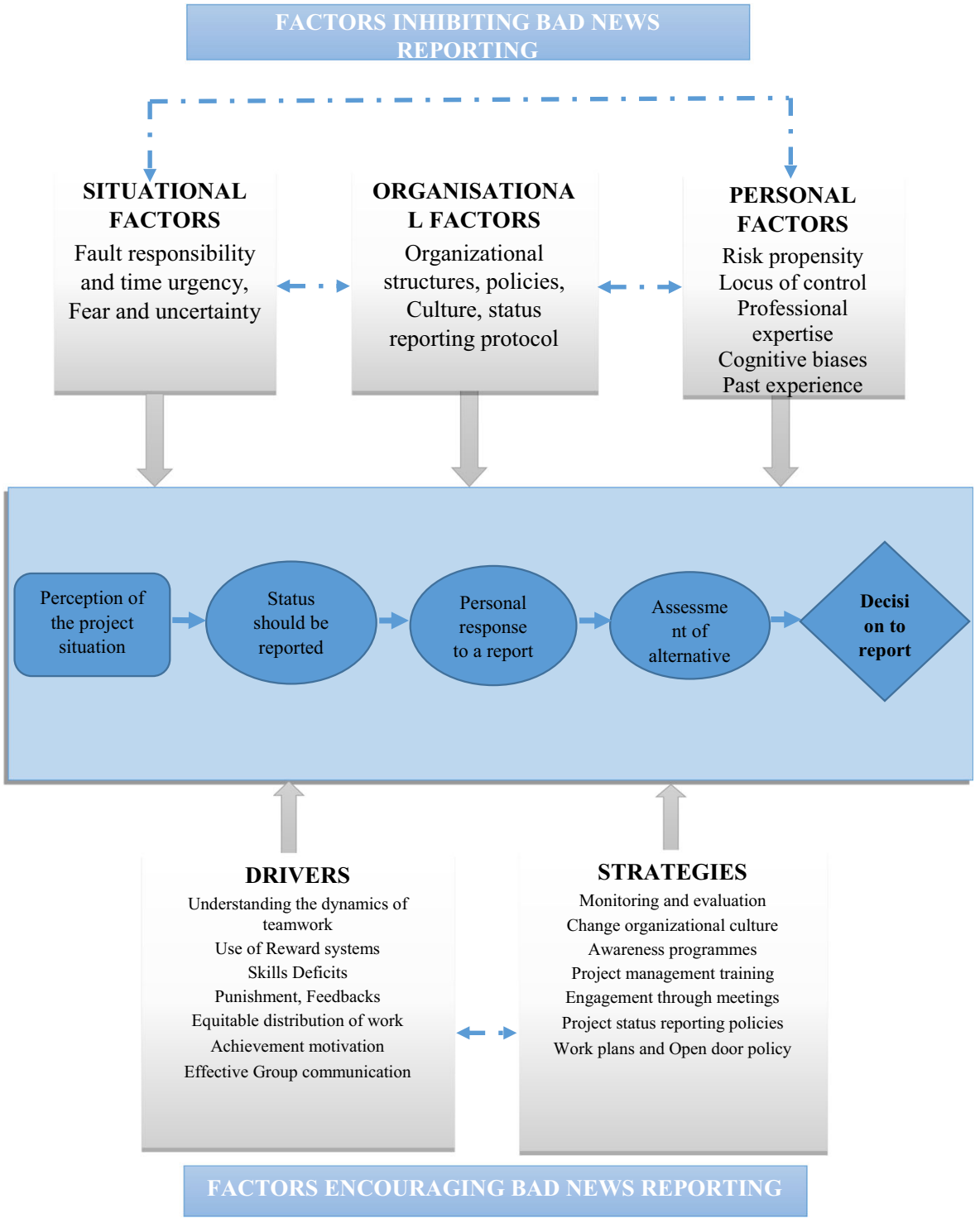

Fig. 2 The proposed framework to manage bad news reporting in state universities in Zimbabwe

dispassionately evaluate the project and therefore report sections and components of the project that may not be performing to expectation is guided by the identified factors. In any case, the responsibility or commitment to make a report has its challenges. The one who has to make the report must assess the potential consequences of making or not making that report, especially where it is going to be a negative report. One may consider the possibility of retaliation such as being labelled a back-stabber by team members who may be found at fault or being ejected from the project team. In this way the decision to report hinges on the individual's judgment of the potential consequences of their action. 
Factors inhibiting BNR were subdivided into organisational factors, situational factors, and personal factors as shown in the upper part of the framework. Organisational factors include organizational structures, policies, culture, and the status reporting protocol. Situational factors include fault responsibility and time urgency, fear, and uncertainty. Personal factors include risk propensity, locus of control, professional expertise, cognitive biases, and past experience. The research findings show that these three factors are interrelated. This means that factors from one category are interlocked with factors belonging to another category. For instance, the lack of policies that formalise BNR can breed in project team members a sense of fear and uncertainty. They may become uncertain about what would befall them if they decided to report any negative news about the software project. Arrows to and from each of the general factors represent the interaction of these factors in the framework.

Factors encouraging BNR were categorized into two, namely drivers and strategies. Drivers of BNR motivate individuals to report bad news about a software project and they include understanding the dynamics of teamwork, use of reward systems, skills deficits, punishment, feedbacks, equitable distribution of work, achievement motivation, and effective group communication. Drivers may be influenced by the behaviour of individuals in the project team. For example, not every team member who fails to report bad news may be subjected to punishment or penalisation. For instance, first offenders may be absolved yet those who are habitually reluctant to report bad news may be liable for punishment. Thus, as shown by the arrow that feeds into Mark and Keil's model, drivers positively interact with an individual's perception to trigger reporting behaviour for the bad news. The drivers that trigger BNR in software project team members are presented on the lower-left section of the framework.

Strategies are plans of action that the organisation crafts at a macro level to achieve long-term aims. Thus, strategies are by nature long-term actions implemented at the organisational level or departmental level. Strategies include Monitoring and evaluation, change organizational culture, awareness programmes, project management training, engagement through meetings, project status reporting policies, work plans, and open-door policy. Strategies are shown on the lower right side of the model as positive factors encouraging BNR.

Demonstration and evaluation steps are an essential part of design science. To ensure rigour and soundness the framework was subject to expert review. The framework was reviewed by four experts who were chosen based on their knowledge and experience. The four experts consisted of academics and practitioners. Two academics hold doctorates in Information Technology and Software Engineering. The first academic reviewer worked for 10 years as n ICT director of a university before joining academia. The practitioners with a combined experience of 30 years of experience in developing and implementing university systems. These practitioners were drawn from universities that were not selected to take part in this study. The reviewer evaluated the framework for relevance, usability, usefulness, and applicability in the problem domain and 
context and gave feedback. The reviewer's comments were incorporated in the refinement of the framework.

\section{Conclusion and implications}

Within universities, the motive not to report bad news on a software project is quite prevalent. Reluctance to report bad news may be a decision that has serious consequences for individuals and the institutions at large. Since project team members are reluctant to report bad news, positive information is likely to be conveyed quickly and liberally up the organisational structure compared to undesirable information. This means that a large chunk of undesirable information, which may be important to the organisation, ends up failing to reach the ears of senior management. Such information asymmetry has adverse effects on how management decisions are made. It means that superiors are bound to make their decisions, not from a position of full information as quietness or that elective silence about important issues can compromise the detection of errors as well as decision making around those faults.

This study recommends the establishment of clear channels of communication to manage BNR. This is in sync with Milliken et al. (2003) view that creating formal structures that function outside the traditional organisational hierarchy to convey information regarding glitches is a sound strategy of promoting BNR. However, such structures need to be built on grounds that are appreciative of the nature of problems and fears that may drive project team members' reluctance to raise bad news. This strategy goes a long way in trying to manage BNR on projects. In support of this notion Jung-Chieh et al. (2016) state that individuals with plausible views regarding work improvement but have no motivation or are reluctant to speak out, should liaise with their managers regarding submission of those ideas to superiors who then bring them up for evaluation. There is no doubt that fostering a culture of openness regarding organisational communication can yield positive results in managing BNR on software projects. When carefully managed through formal structures BNR will contribute immensely towards the success of the organisation particularly for the successful delivery of software projects.

The study, to the best of the researchers' knowledge, is the first that has extensively studied the mum effect in Zimbabwean state universities. Therefore, this study has provided empirical evidence from the practitioners' insights, experiences as well as practices on the mum effect. Consequently, this provides foundations for appropriate interventions from educators and administrators. The proposed framework is comprehensive as it deals with factors inhibiting and encouraging BNR. It also includes strategies to minimise reluctance to report and manage bad news in software projects. Furthermore, another practical contribution of this research was to foster and encourage true status reporting on progress on software projects. The policymakers in Zimbabwean state universities and other educational institutions in other countries could use this research to develop sector-specific policies. Future research can extend the 
study by quantifying the cost of failed software projects in Universities, especially in developing countries.

\section{References}

Aronson, Z. H. (2016). Personal style, culture and performance outcomes in projects: What is the role of project leader individual differences? International Journal of Transitions and Innovation Systems, 5(2), 99-121.

Avgerou, C., \& Walsham, G. (2017). Information technology in context: Studies from the perspective of developing countries: Studies from the perspective of developing countries. New York: Routledge.

Beakley, J. E. (2016). Supervisor-subordinate communication: Workplace bullying and the tyrannical mum effect. International Journal of Business and Management, 11(2), 1-21.

Braun, V., \& Clarke, V. (2006). Using thematic analysis in psychology. Qualitative Research in Psychology, 3(2), 77-101.

Chatterjee, S., \& Maji, B. (2018). A bayesian belief network based model for predicting software faults in early phase of software development process. Applied Intelligence, 48(8), 2214-2228.

Dibble, J. L., \& Levine, T. R. (2010). Breaking good and bad news: Direction of the MUM effect and senders' cognitive representations of news valence. Communication Research, 37(5), 703-722.

Eldow, A., Shakir, M., Mohamad Yahya, A., \& Sohail, I. M. (2017). Using information communication technology as a teaching tool in Sudanese governmental universities of Khartoum State. JOIV: International Journal on Informatics Visualization, 1(4), 150-156.

Hevner, A., \& Chatterjee, S. (2010). Design research in information systems: Theory and practice (Integrated series in information systems) (Vol. 22). New York: Springer.

Hevner, A. R., March, S. T., Park, J., \& Ram, S. (2004). Design science in information systems research. MIS Quarterly, 28(1), 75-105.

Jung-Chieh, L., Yih-Chearng, S., \& Chung-Yang, C. (2016). Examining the impacts of organizational culture and top management support of knowledge sharing on the success of software process improvement. Computers in Human Behavior, 54, 462-474.

Keil, M., \& Park, C. (2010). BNR on troubled IT projects: Reassessing the mediating role of responsibility in the basic whistleblowing model. Journal of Systems and Software, 83(11), 2305-2316.

Kliem, R. L., \& Ludin, I. S. (2019). Reducing project risk (1st ed.). London: Routledge.

Korzaan, M., \& Brooks, N. (2015). The Silent Treatment in IT Projects: Gender Differences in Inclinations to Communicate Project Status Information. Journal of Information Systems Applied Research, 8(1), 19-30. http://jisar.org/2015-8/N1/JISARv8n1.pdf. Accessed 17 Feb 2020.

McBride, M. (2016). Project management basics. Berkeley: Apress.

Milliken, F. J., Morrison, E. W., \& Hewlin, P. F. (2003). An exploratory study of employee silence: Issues that employees don't communicate upward and why. Journal of Management Studies, 40(6), 1453-1476.

Natovich, J., Zeev, D., \& Natovich, R. (2013). Positive psychology in information technology project management: The case of BNR. Information Technology Project Management (IJITPM), 4(4), 35-50.

Padhi, S., \& Sumita, M. (2017). Effect of honesty as an ethical value on software project performance: A grounded theory approach. Journal of Human Values, 23(2), 92-105.

Peffers, K., Tuunanen, T., Rothenberger, M. A., \& Chatterjee, S. (2007). A design science research methodology for information systems research. Journal of Management Information Systems, 24(3), $45-77$.

Ramingwong, S., \& Ramingwong, L. (2013). A tale behind mum effect. International Journal of Information Systems and Project Management, 1(3), 47-58.

Shaya, O. (2013). Status reporting in IT projects. Göttingen:Universität Göttingen.

Smith, J. H., \& Keil, M. (2003). The reluctance to report bad news on troubled software projects: A theoretical model. Information Systems Journal, 13(1), 69-95.

Teklemariam, M. A., \& Mnkandla, E. (2017). Software project risk management practices in Ethiopia. The Electronic Journal of Information Systems in Developing Countries, 79(7), 1-14.

Tesser, A., \& Rosen, S. (1975). The reluctance to transmit bad news. Advances in experimental social psychology. Advances in Experimental Social Psychology, 8, 193-232.

Veletsianos, G., \& Kimmons, R. (2016). Scholars in an increasingly open and digital world: How do education professors and students use twitter? The Internet and Higher Education, 30, 1-10. 
von Solms, B. (2006). What every Vice-Chancellor and Council Members should know about the use of ICT. In Proceedings of the conference on information technology in tertiary education, Pretoria, South Africa, 18-20 September 2006. The Pennsylvania State University: CiteSeerX.

Wixom, B. H., \& Watson, H. J. (2001). An empirical investigation of the factors affecting data warehousing success. MIS Quarterly, 25(1), 17-41.

Zanin, A. C., Bisel, R. S., \& Adame, 1. A. (2016). Supervisor moral talk contagion and trust-in-supervisor: Mitigating the workplace moral mum effect. Management Communication Quarterly, 30(2), 147-163.

Publisher's note Springer Nature remains neutral with regard to jurisdictional claims in published maps and institutional affiliations. 